\title{
PERCEPCIÓN DE LAS ENFERMERAS SOBRE EL USO DE SUS REGISTROS PARA GARANTIZAR LA CONTINUIDAD DEL CUIDADO
}

\section{NURSES' PERCEPTION OF THE USE OF THEIR RECORDS TO ENSURE CONTINUITY OF CARE}

Clarivel De Fátima Soza Diaz (1), Asunción Carmen Luz Bazán Sánchez (2) Rosa Jeuna Diaz Manchay (3)

Licenciada en Enfermería. Escuela de Enfermería de la Universidad Católica Santo Toribio de Mogrovejo-

Chiclayo, Perú. E-mail: clafasodi@gmail.com;

ORCID iD: https://orcid.org/0000-0002-7578-6437

Maestra en Ciencias de Enfermería. Docente de la Escuela de Postgrado de la Universidad Católica Santo Toribio de Mogrovejo- Chiclayo, Perú. E-mail: camuchabs@hotmail.com

ORCID iD: https://orcid.org/0000-0002-9501-6747

Doctora en Ciencias de Enfermería. Coordinadora de Asuntos Académicos de la Escuela de Enfermería de la Universidad Católica Santo Toribio de Mogrovejo-Chiclayo, Perú. E-mail: rdiaz@usat.edu.pe; ORCID iD: https://orcid.org/0000-0002-2333-7963

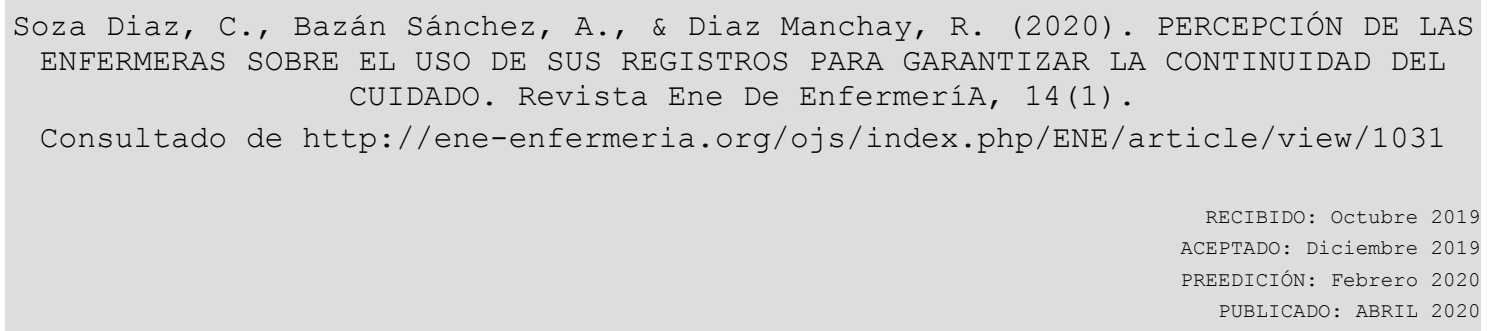




\section{Resumen}

Introducción: Los registros de enfermería son documentos que avalan la calidad y continuidad del cuidado, mejora la comunicación y evita errores durante el cuidado. Objetivo: Describir la percepción de las enfermeras sobre el uso de sus registros para garantizar la continuidad del cuidado en el servicio de medicina interna de un hospital público. Métodos: Investigación cualitativa descriptiva, la muestra fueron 12 enfermeras, se usó la entrevista semiestructura. Resultados: Utilidad e importancia para el equipo de salud; Limitantes e implicancias éticolegales; Necesidad de registros estandarizados e informatizados. Conclusión: Los registros de enfermería llenados con veracidad garantizan la continuidad del cuidado porque son fuente de información para los profesionales del equipo de salud; pero, existen limitantes como la falta de tiempo, la sobrecarga laboral por que se omiten datos que pueden incurrir en implicancias ético-legales, por lo que reconocen la necesidad de formatos estandarizados y digitalizados.

Palabras clave: Percepción, Registros de Enfermería, Atención de enfermería.

\section{Abstract}

Introduction: Nursing records are documents that guarantee the quality and continuity of care, improve communication and avoid errors during care. Objective: To describe the perception of nurses about the use of their records to guarantee continuity of care in the internal medicine service of a public hospital. Methods: Qualitative descriptive research, the sample was 12 nurses, the semistructured interview was used. Results: Usefulness and importance for the health team; Limitations and ethical-legal implications; Need for standardized and computerized records. Conclusion: Nursing records filled with truth guarantee continuity of care because they are a source of information for health team professionals; However, there are limitations such as lack of time, work overload due to the omission of data that may incur ethicallegal implications, which is why they recognize the need for standardized and digitized formats.

Keywords: Perception, Nursing Records, Nursing Care. 


\section{INTRODUCCIÓN}

En la profesión de enfermería, la práctica de registrar o documentar se remonta al tiempo de la máxima referente de enfermería, Florence Nightingale, quien al ver las condiciones tan indignas en las que eran atendidos los soldados y las operaciones poco higiénicas, entendió que enfermedades como el cólera y el tifus invadieran los hospitales, entonces comprendió que era necesario recolectar datos y organizarlos en un sistema para llevar el registro correcto, situación que más adelante serviría para mejorar los hospitales militares y sobre todo, para calcular la tasa de mortalidad del hospital $(1,2,3)$. Desde entonces los registros de enfermería fueron de gran valor, ya que no solo sirvieron para registrar hechos, sino que estos ayudaron a tomar medidas correctivas, normativas y continuar el cuidado en bien de las personas.

De Marinis et al (4) afirman que, en el historial de enfermería, los registros son documentos propios de la profesión y básicos de la historia clínica. En ellos se recolectan datos de las acciones emprendidas, las observadas y de la información obtenida del paciente y la familia, con el fin de optimizar la comunicación entre los miembros del equipo de salud y por consiguiente garantizar una continuidad en el cuidado; además son un respaldo legal frente a posibles demandas y a la vez permiten evaluar retrospectivamente la calidad de los cuidados; sin dejar de reconocer, que también encierran el nivel de práctica ética en el cuidado. Chávez (5) determinó la importancia de los registros de enfermería en los procesos asistenciales de los pacientes; sin embargo, no son llevados adecuadamente y la falta de normativa institucional rezaga su uso. Mota, Olivera y Da Silva (6) concluyeron que los registros de enfermería son cifrados, ilegibles, presentan errores ortográficos, espacios en blanco, y uso indiscriminado de abreviaturas y siglas.

Por otro lado, el paciente puede solicitar acceso a esta información como parte de los derechos que le asisten, para ello, estos registros son fuente de información importante y deben tener la garantía de ser reflejo de las intervenciones de enfermería Además, si existiera algún tipo de reclamo por la atención, los registros son la evidencia del cuidado brindado y del cumplimiento de las indicaciones, aspecto legal que es importante tener en cuenta, basado en la Norma Técnica de Salud para la Gestión de la Historia Clínica (7), la cual establece el manejo estandarizado del contenido básico a ser registrado en la Historia Clínica, en relación con el conjunto de prestaciones que se oferta y recibe el usuario de salud, respetando los aspectos lega- 
les y administrativos del proceso de atención de salud. A pesar de que esta normado, no se efectiviza su cumplimiento en los establecimientos de salud.

Al respecto, en el servicio de Medicina Interna de un Hospital local en Chiclayo- Perú, los registros de enfermería son realizados de manera manual en los diferentes formatos como las anotaciones de enfermería, hoja grafica de signos vitales, hoja de balance hídrico, entre otros formatos que son utilizados como medios de información por el equipo de salud, con la finalidad de valorar la evolución, si se efectivizó las indicaciones, y por ende garantizar la continuidad del cuidado. Sin embargo, veces se observa omisiones, letras ilegibles, entre otros aspectos que evaden la calidad requerida y que podrían incurrir en problemas ético-legales.

\section{OBJetivo}

Objetivo: Describir la percepción de las enfermeras sobre el uso de sus registros para garantizar la continuidad del cuidado en el servicio de medicina interna de un hospital público en la ciudad de Chiclayo, Perú.

\section{MÉTODOS}

La investigación fue de tipo cualitativa descriptiva (8). La muestra lo conformaron 12 enfermeras que trabajan en el servicio de medicina interna de un hospital público de Chiclayo, Perú. El muestreo fue no probabilístico, por conveniencia (9) y el tamaño de la muestra se determinó por criterio de saturación y redundancia. Para proteger su identidad y favorecer su participación se usó códigos: ENF1... ENF12; se les informó que la entrevista era grabada, con una grabadora de voz, para luego ser transcritas y posteriormente presentados a cada uno de los participantes para corroborar que lo expresado es lo correcto y para agregar algún dato en algunos de los relatos proporcionados.

Los datos se recolectaron a través de la entrevista semiestructurada (10), que fue elaborada por la investigadora principal, a partir de los antecedentes y bases teóricas, y validada mediante juicio de expertos. Después de obtenida la aprobación del proyecto por el Comité de Ética en Investigación de la Facultad de Medicina con la Universidad Católica Santo Toribio de Mogrovejo, y del permiso para ejecución de las autoridades del hospital, se procedió a ubicar a las enfermeras asistenciales que intervinieron en la investigación; previa firma del Consentimiento Informado se realizaron las 
entrevistas entre octubre y noviembre del 2018, en la oficina de la jefatura de Enfermería del servicio de medicina interna del nosocomio para favorecer la privacidad y evitar distracciones; se ejecutaron en horario fuera de turno, con una duración de aproximadamente treinta minutos.

Para el análisis de los datos se utilizó el análisis de contenido (11), ya que se encarga de estudiar material cualitativo, buscando la mejor compresión de una comunicación y la examina a profundidad. Esto se realizó respetando sus tres etapas: En el preanálisis se realizó la revisión de los datos obtenidos realizando la lectura y relectura de los discursos, para seleccionar la información más relevante. En la codificación se realizó el proceso de clasificación de la información en base a ideas y conceptos adquiridos mediante el propio material de estudio y se confrontó con el marco teórico. Los datos brutos fueron transformados sistemáticamente a datos que fueron útiles para identificar unidades significativas, las cuales fueron identificadas por el método de coloreo y fueron relacionadas con los objetivos de la investigación. En la categorización se organizaron y clasificaron las unidades obtenidas en base a criterios de diferenciación; se agrupó las unidades por similitudes en cuanto a su significado, contrastando la realidad con la literatura, logrando así la categorización de los elementos en relación con los objetivos de la investigación.

Durante todo el desarrollo de la investigación se aplicaron los principios de la ética personalista (12), los sujetos de estudio no fueron sometidos a experimentación, no sufrieron daño físico ni emocional, eligieron de manera voluntaria participar de la investigación mediante el consentimiento informado, se protegió su identidad. Además, se tuvo en cuenta los criterios de rigor científico (9) como la credibilidad, transferibilidad y confirmabilidad.

\section{Resultados}

\section{A: Utilidad e importancia de los} registros de enfermería para el equipo de salud.

Los registros de enfermería conforman la evidencia escrita de los cuidados otorgados al paciente, a su vez, son por excelencia un medio de comunicación y coordinación que facilita el trabajo entre los miembros del equipo de salud, por tanto, su realización correcta permite avalar la continuidad de los cuidados y la seguridad del paciente.

“...los registros de enfermería sirven para monitorizar y registrar los procedimientos realizados a los pacien- 
tes, son una guía para poder seguir con la continuidad y que quede plasmado mi trabajo" (ENF4)

“...mediante los registros, evidencias sobre: el tratamiento administrado, el balance hídrico, el Kárdex, las actividades propias del turno y continuar lo que viene, para que, si se ha hecho algo, ya no lo repitas, porque a veces no registras, te entra la duda y tienes que llamar, a la colega que salió de turno..." (ENF5) “...los registros son útiles para todo el equipo de salud, son algo que deberíamos hacer bien para entendernos, en el caso de los médicos, es importante porque ellos se guían de los registros (Anotaciones de enfermería, ingresos, egresos, grafica de controles vitales etc.) a la hora de pasar la visita, para dar prescripciones de tratamiento..." (ENF1)

“...los médicos en varias oportunidades hacen uso de los registros, siempre en la visita médica se fijan en algunos, como la hoja gráfica de signos vitales, balance hídrico, las hojas de escalas de riesgo (Downton y Norton), y revisan la curva febril porque nosotras lo manejamos. En las notas ven lo que hicimos con el paciente...todos les sirven para evaluar al paciente y plantear la terapéutica" (ENF3)
Además, sus registros facilitan la comunicación con todo el equipo de salud y guían las decisiones terapéuticas centradas en el paciente, otorgándole una connotación de apoyo para el personal médico y contribuye a brindar un cuidado de calidad, que mantenga la continuidad del cuidado.

\section{B: Limitantes e implicancias} ético-legales en los registros de enfermería

Las enfermeras perciben que su principal limitante para realizar correctamente los registros es la falta de tiempo por la demanda de procedimientos que realizan, se dedican a los cuidados quedando poco tiempo para la labor administrativa, por ello solo registran de los pacientes más delicados.

“...no nos alcanza el tiempo para registrar todo lo que hacemos, solo registramos lo que hacemos con los pacientes más delicados..." (ENF1)

“...las seis horas de turno no son suficientes para completar los registros porque se me va en procedimientos, tratamiento, por tanto, registro lo más esencial y lo demás si lo informo verbalmente a la colega de turno..." (ENF11) 
Por otro lado, expresan las implicancias ético-legales en los registros de enfermería.

“...cuando nos formaron, nos enseñaron que parte de la ética es registrar con veracidad lo que se hace en el paciente y también la institución lo exige y tienes que cumplir responsablemente... hay que registrar y hacerlo bien..." (ENF8)

“...es importante que haya concordancia en lo que tú haces con lo que estás registrando, porque de repente puedes decidir registrar que hiciste algún procedimiento $y$ en realidad no lo has hecho, hay que hacer lo que es correcto ... son un respaldo legal ante cualquier situación, como por ejemplo cuando llamas al médico de turno por una emergencia y te da indicaciones por teléfono... si ocurre algo a quien acusan directamente es a la enfermera porque ella es la que hace efectivas las prescripciones médicas, hasta telefónicas...por eso yo pongo en la historia clínica que recibí la indicación telefónica ...” (ENF9)

$$
\text { “... ahora hay más exigencia para }
$$
hacer correctamente los registros hasta mandan memorándums... además ante un reclamo o denuncia, si todos los registros de enfermería están bien hechos, son nuestro respaldo" (ENF12)
Los registros de enfermería pueden ser un buen (o mal) reflejo del estándar de atención que se brinda a los pacientes. Es como el sello distintivo de cada enfermera, pero si no están escritos conforme a las normas estipuladas, puede llevar, no solo a dudas sobre la calidad del trabajo de la enfermera, sino, sobre todo, podría verse implicada en problemas ético- legales y ser sancionada.

\section{C: Necesidad de registros de} enfermería estandarizados e informatizados

Las enfermeras manifiestan que son varios los registros que utilizan y que los llenan de forma manual ello implican poca uniformidad y gran inversión de tiempo, por lo que consideran necesario que se implementen registros estandarizados y en el mejor de los casos la informatización de estos.

“...me gustaría que fuera como en otras unidades, en sus registros ahí no más te detallan, como una lista de comprobación y algo para anotar algunas cosas subjetivas" (ENF4)

“...los registros deben ser más prácticos, como la hoja gráfica de Signos Vitales, se podría agregar otros ítems para marcar...ya no habría pretexto para no cumplir" (ENF9) 
“...cuando los registros no están ingresados al sistema de Gestión hospitalaria en la computadora, pues se pierde nuestro trabajo... hacemos bastante $y$ no está registrado... sería muy útil poner en práctica lo que ya se está dando en otros países, que todo sea informatizado" (ENF10)

Ellas muestran interés en que los registros de enfermería sean más prácticos, que detallen toda la información necesaria para garantizar la continuidad del cuidado, y a su vez, que las ayude a disminuir la carga laboral. Además, demuestran su interés por un registro informatizado que sea ingresado al Sistema Informático de Gestión Hospitalaria, ya que así se podría evidenciar todo lo efectuado por el profesional de enfermería, de una manera más fácil.

\section{Discusión}

Los hallazgos de esta investigación reflejan que la importancia de los registros de enfermería radica en que facilitan el intercambio de información, y sirven como guía para el equipo de salud pues facilita la toma de decisiones, siendo su propósito proporcionar datos sobre la atención brindada, lo cual garantiza la comunicación escrita y la continuidad del cuidado.
Los datos encontrados en esta investigación se corroboran con el estudio de López-Cocotle, Moreno-Monsiváis, y Saavedra-Vélez (13), pues aseveran que los registros de enfermería evidencian el rol autónomo de la disciplina y así, diferencian sus actividades del resto de los profesionales del equipo multidisciplinario de salud. Así también, SuárezVilla (14) concluye que los registros de enfermería son parte esencial de la historia clínica, los cuales almacenan información del paciente, lo cual sirve para su beneficio, permitiendo la continuidad de los cuidados. Por ello, los registros deben consignar los cuidados enfermeros realizados en el turno con cada paciente, como para facilitar el posible seguimiento por parte de otros profesionales de enfermería; por consiguiente, está obligado a extremar el rigor de su contenido, es decir registrar lo que se planificó, dijo, se hizo y lo que quedó pendiente acerca del paciente; de ahí se deriva su importancia principal en describir el problema del paciente y orientar la terapéutica, para así garantizar la continuidad del cuidado tal como lo manifiestan los estudios de Torrecilla (15) y, López, Moreno y Saavedra (16).

En este estudio los registros de enfermería conforman la principal vía de comunicación importante para el equipo de salud involucrado con el paciente. 
Como manifiestan Ochoa, Pace, Dos

Santos (17) los registros de enfermería, facilitan la coordinación y continuidad de la planificación de la atención de salud, y cada profesión contribuye con el cuidado a los pacientes. En este sentido debe entenderse que estos registros no solamente tienen importancia y utilidad para la enfermera, también es útil e importante para el equipo de salud, sobre todo para, con quienes más se coordina la atención de salud y los cuidados enfermeros: los médicos, quienes revisan las anotaciones de enfermería y otros registros, en el momento de pasar visita médica. Lo dicho anteriormente es similar al estudio de Fernández, Ruydiaz y Del Toro (18), quienes señalan que un registro estructurado correctamente, da confianza, respeto y comprensión al equipo de salud, y expresa una interacción recíproca y precisa del hecho de cuidar.

Pero si los registros no son llenados correctamente puede conllevar a errores en la seguridad del cuidado. En el estudio evidencian que la falta de tiempo coarta el cumplimiento de todos sus registros, dado a que las múltiples tareas como procedimientos y tratamientos que realizan en las seis horas del turno, tiempo insuficiente que les limita a solo registrar lo más relevante, dejando lo otro para informar verbalmente durante el reporte de enfermería, poniendo en riesgo la continuidad del cuidado. Al respecto Ríos (19), coincide con lo vertido, indicando que uno de los principales factores atribuidos por el personal de enfermería asociados al incumplimiento del correcto llenado de sus registros es el exceso de trabajo, que limita el tiempo para registrar las actividades realizadas. Aspectos que conllevan a problemas ético-legales, exponiéndose a problemas con sus pares, la ley o con la institución. Siendo necesario que se actúe cumpliendo los protocolos y normativas vigentes, aplicando los postulados de la conciencia moral (20); puesto que según León, Manzanera, López y Gonzáles (21), no se debe olvidar considerar a los registros de enfermería como documentos obligatorios desde el marco jurídico y moral, pues constituyen el testimonio documental de las intervenciones de enfermería y a su vez, si no se registra la actividad realizada es imposible constatar lo que se aporta en bien del paciente ante posibles reclamaciones.

En el contexto del estudio, las prescripciones telefónicas son bastante frecuentes, en caso de molestias agudas imprevistas, estas se regularizan, pero son un inconveniente para proceder al tratamiento; preferentemente no se deben tomar órdenes verbales o telefónicas, pues se puede incurrir en problemas legales. La prescripción médica es un 
acto complejo, que requiere un gran sentido de responsabilidad y una actitud ética, el prescriptor asume la responsabilidad legal por las implicancias de la prescripción (22). Aunque la enfermera es la ejecutora de estas prescripciones, por lo tanto, también está implicada.

En este estudio los registros son realizados de forma manual, lo que demanda mayor tiempo, por ello manifiestan una necesidad latente de implementar registros estandarizados e informatizados. Los registros estandarizados, tienen un patrón determinado, son sencillos y claros, permiten ahorrar tiempo, esto se traducirá en una mejora de su eficiencia como servicio y limitaría los errores (23). Los registros electrónicos de enfermería son la nueva metodología en donde se puede evidenciar el cuidado brindado a los pacientes, siendo un elemento de defensa legal ante posibles procesos disciplinarios entre otras aplicaciones (24); lo cual coincide con la investigación de Nestares y Sayas (25), quienes concluyeron que el registro electrónico de enfermería muestra mayor efectividad para la continuidad del cuidado, en comparación con el registros escrito, así también aportan que con el avance de la tecnología, enfermería debe permanecer a la vanguardia, con el fin de beneficiar al paciente. Finalmente, las enfermeras consideran que, los registros de enfer- mería, al no ser ingresados al Sistema informático de gestión hospitalaria, se pierden y es como si no hubieran realizado ninguna actividad, lo cual demuestra la necesidad de visibilidad de sus registros y que sea más práctico de realizar con ayuda de la tecnología.

\section{CONCLUSiones}

Las enfermeras del estudio establecen que los registros de enfermería garantizan la continuidad del cuidado porque son fuente de comunicación e información de los cuidados brindados, lo cual está relacionado a la responsabilidad ética y legal de sus cuidados. Además, son documentos de consulta entre las mismas enfermeras al cambiar de turno y para el resto del personal de salud, principalmente en la visita médica, donde los datos sirven de sustento para completar su evaluación y prescribir lo mejor para el paciente.

Por otro lado, las enfermeras reconocen al tiempo y la demanda de procedimientos, como las principales limitantes en el cumplimiento de los registros de enfermería, así también, hacen hincapié, que no es el número de pacientes, sino la complejidad y número de tratamientos, cuidados y procedimientos que ellos necesitan. Reconocen que estos documentos tienen connotación ética legal, pero cuando reciben prescripción 
médica por vía telefónica en alguna situación especial, lo deben registrar con el fin de salvaguardar la seguridad del paciente y de ellas mismas frente a cualquier tipo de reclamo. Asimismo, aspiran a que sean estandarizados y tengan un programa informático que registre todas sus actividades, para que sirvan como evidencia de su trabajo, con lo que lograrán mayor visibilidad de sus cuidados. 


\section{BIBLIOGRAFÍA}

1. Young $P$, Hortis V, Chambi M, Finn B. Actualidad evangélica: Florence Nightingale (1820-1910), "madre" de la enfermería moderna [Internet]. Buenos Aires: Hospital Británico de Buenos Aires; 2014 [Consultado 5 Abril 2018]. Disponible en: https://www.actualidadevangelica.es/index.php? option=com_content\&view=article\&id=7448:florence-nightingale-1820-1910-madre-de-la-enfermeriamoderna\&catid $=43$ :destacados

2. Amaro MC. Florence Nightingale, la primera gran teórica de enfermería. Rev Cubana Enfermer [Internet]. 2004 Diciembre [Consultado 5 Abril 2018]; 20(3). Disponible en: http://scielo.sld.cu/

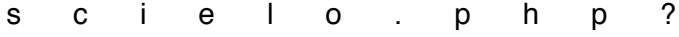
script=sci_arttext\&pid=S0864-0319200400030000 $9 \& \operatorname{lng}=\mathrm{es}$.

3. Palmer IS. Florence Nightingale: reformer, reactionary, researcher. Nur Res [Internet]. 1977 Abril [Consultado 5 Abril 2018]; 26(2):84-9. Disponible en: https://www.ncbi.nlm.nih.gov/pubmed/320564

4. De Marinis MG, Piredda M, Pascarella MC, Vincenzi B, Spiga F, Tartaglini D, Alvaro R, Matarese $M$. If it is not recorded, it has not been done. J Clin Nurse [Internet] 2010 Junio [Consultado 5 Abril 2018]; 19 (11-12):1544-52. Disponible en: https:// www.ncbi.nlm.nih.gov/pubmed/20438599

5. Chávez D. Utilización de los registros clínicos de Enfermería por el equipo de salud en los procesos asistenciales de los pacientes del Servicio de Medicina Interna del Hospital Pablo Arturo Suarez [Tesis de Licenciatura en internet]. Quito: Pontificia Universidad Católica del Ecuador; 2013 [Consultado 16 de abril 2018]. 132p. Disponible en: http:// repositorio.puce.edu.ec/bitstream/handle/ 22000/7228/8.14.001708.pdf?sequence=4\&isAllowed=y

6. Mota M, Oliveira S, Da Silva P. Registros de enfermagem: reflexões sobre o cotidiano do cuidar. ABCS Health Sci. [Internet] 2017 Julio [Consultado 13 Junio 2018]; 42(3):161-165. Disponible en: ht t p://docs.bvsalud.org/biblioref/ 2017/12/876234/42abcs161.pdf

7. Ministerio de Salud. Norma Técnica de Salud para la Gestión de la Historia Clínica. [Internet] Lima; 2018. 123p. [Consultado 16 de abril 2018]. Disponible en: ftp://ftp2.minsa.gob.pe/normaslegales/ 2018/R.M_214-2018-MINSA2.pdf

8. Katayama R. Introducción a la Investigación Cualitativa. Lima: Fondo Editorial de UIGV; 2014.126p.

9. Hernández R, Fernández C, Baptista P. Metodología de la Investigación. 6ta Edición. México D.F.: Mc Graw Hill Education; 2014. 599p.

10.Cifuentes R. Diseño de proyectos de Investigación Cualitativa. Buenos Aires: Noveduc; 2014.167p.
11.Cassuli M, Fustinoni S. Pesquisa Qualitativa em enfermagem. Sao Paulo: Editora Médica Paulista; 2006. $155 \mathrm{p}$.

12.Sgreccia E. Manual de bioética. I: Fundamentos y ética biomédica. Biblioteca de Autores Cristianos: Madrid -España; 2009. 972 p.

13.López-Cocotle J.J, Moreno-Monsiváis M.G., Saavedra-Vélez C.H.. Construcción y validación de un registro clínico para la atención asistencial de enfermería. Enferm. univ [revista en Internet]. 2017 Dic [Consultado 01 de Abril 2019]; 14( 4 ): 293-300. Disponible en: http://www.scielo.org.mx/ $\mathrm{s}$ c i e l o . p h p ? script=sci_abstract\&pid=S1665-706320170004002 93\&lng=es\&nrm=iso

14.Suárez-Villa M. Registros de Enfermería como Evidencia del Cuidado. Cienc. innov. salud. [Internet]. 2013; 1 (2):126 - 133. [Consultado el 2 setiembre del 2019]. Disponible en: http://revistas.unisimon.edu.co/index.php/innovacionsalud/article/ view/59

15. Torrecilla E. Necesidad de registrar en la profesión enfermera. [Tesis de Maestría en Internet]. Catalunya: Universitat Internacional de Catalunya; 2015. [Consultado 30 Octubre 2018]. 52p. Disponible en: https://www.recercat.cat/bitstream/handle/ 2072/252220/TFG_Estefan\%C3\%ADa_TorreciIla_Cilleros.pdf?sequence $=1$

16. López J, Moreno M, Saavedra C. La importancia del registro clínico de enfermería: un acercamiento cualitativo. Nure Inv. [Internet]. 2018 Abr-May [Consultado 30 octubre 2018]; 15(93). Disponible en: https://www.researchgate.net/publication/ 324261212_La_importancia_del_registro_clinico_de_enfermeria_un_acercamiento_cualitativo

17. Ochoa K, Pace A, Dos Santos C. Análise Retrospectiva Dos Registros De Enfermagem Em Uma Unidade Especializada. Rev Latino - Am Enfermagem. [Internet]. 2003 Mar- Abr [Consultado 30 octubre 2018]; 11(2):184-91. Disponible en: http:// www.scielo.br/pdf/rlae/v11n2/v11n2a07

18.Fernández S, Ruydiaz K, Del Toro M. Notas de enfermería: una mirada a su calidad. Salud, Barranquilla [Internet]. 2016 Mayo [Consultado el 07 de Setiembre del 2019]; 32 (2): 337-345. Disponible en: http://www.scielo.org.co/scielo.php? script=sci_arttext\&pid=S0120-5552201600020001 $5 \& \operatorname{lng}=e n$.

19.Ríos L. Factores asociados al incumplimiento del llenado correcto de las notas de enfermería en el servicio de medicina del Hospital Amazónico de Yarinacocha- Pucallpa, julio - diciembre 2017. [Tesis de Licenciatura en Internet]. Tarapoto: Universidad Nacional de San Martín; 2018. [Consultado 01 Abril 2019]. 89p. Disponible en: http://repositorio.unsm.edu.pe/handle/UNSM/2809 
20.Valderrama A, López R. Conciencia moral: ampliando su aplicación en salud. Aspectos teóricos y prácticos de los juicios de conciencia en Chile. Acta bioeth. [Internet]. 2011 Nov [Consultado el 14 de marzo 2019]; 17( 2 ): 179-188. Disponible en: https://scielo.conicyt.cl/scielo.php?script=sci_arttext\&pid=S1726-569X2011000200004

21.León J, Manzanera J, López P, González L. Dudas de enfermería en el manejo de la historia clínica. Aspectos jurídicos. Enf Global [Internet]. 2010, 9 (2) 1-14. [Consultado el 7 de septiembre del 2019]. Disponible en: https://revistas.um.es/eglobal/article/view/107331

22.MINSA. Manual de Buenas Prácticas de Prescripción / Ministerio de Salud. Dirección General de Medicamentos, Insumos y Drogas. Lima: Ministerio de Salud, 2005. [Consultado 14 de marzo 2019]. Disponible en: http://bvs.minsa.gob.pe/local/MINSA/1431.pdf

23.KYOCERA. La estandarización de procesos, una ventaja competitiva. [Internet] 2017. [Consultado 14 Mayo 2019]. Disponible en: https://smarterworkspaces.kyocera.es/blog/la-estandarizacionprocesos-una-ventaja-competitiva/

24.Figueroa C, Mejía K, Santa Cruz K. Calidad de los registros electrónicos de enfermería y los factores que influyen en la Unidad de Cuidado Intensivo del Hospital Guillermo Kaelin de la Fuente [Tesis de Licenciatura en internet]. Perú: Universidad Peruana Cayetano Heredia; 2016 [Consultado 16 de junio 2019]. 51p. Disponible en: http://repositorio.upch.edu.pe/handle/upch/595

25. Nestares J, Sayas C. Efectividad del registro electrónico comparado con el registro manual para la continuidad del cuidado de enfermería en la unidad de cuidados intensivos. [trabajo final de especialidad en Internet]. Perú: Universidad Privada Norbert Wiener, 2018 [Consultado 06 de Setiembre de 2019] 44 p. Disponible en: http://repositorio.uwiener.edu.pe/handle/123456789/2519 